\title{
dspace.vutbr.cz
}

\section{Comparative Study of Op-Amp-based Integrators Suitable for Fractional-Order Controller Design}

\author{
HERENCSÁR, N.; KARTCl, A.; YILDIZ, H. A.; ŠOTNER, R.; \\ DVOŘÁK, J.; KUBÁNEK, D.; JEŘŔBEK, J.; KOTON, J.
}

\begin{abstract}
Proceedings of the 2019 42nd International Conference on Telecommunications and Signal Processing (TSP), pp. 740-743
\end{abstract}

elSBN: 978-1-7281-1864-2

DOl: http://dx.doi.org/10.1109/TSP.2019.8768843

Accepted manuscript

(C2019 IEEE. Personal use of this material is permitted. Permission from IEEE must be obtained for all other uses, in any current or future media, including reprinting/republishing this material for advertising or promotional purposes, creating new collective works, for resale or redistribution to servers or lists, or reuse of any copyrighted component of this work in other works. HERENCSÁR, N.; KARTCI, A.; YILDIZ, H. A.; ŠOTNER, R.; DVOŘÁK, J.; KUBÁNEK, D.; JEŘÁBEK, J.; KOTON, J., "Comparative Study of Op-Amp-based Integrators Suitable for Fractional-Order Controller Design", Proceedings of the 2019 42nd International Conference on Telecommunications and Signal Processing (TSP), pp. 740-743-364, 2019. DOl: 10.1109/TSP.2019.8768843. Final version is available at https://ieeexplore.ieee.org/document/8768843 


\title{
Comparative Study of Op-Amp-based Integrators Suitable for Fractional-Order Controller Design
}

\author{
Norbert Herencsar ${ }^{*}$, Aslihan Kartci ${ }^{* \dagger}$, Hacer A. Yildiz ${ }^{\ddagger}$, Roman Sotner ${ }^{* \dagger}$, \\ Jan Dvorak ${ }^{*}$, David Kubanek ${ }^{*}$, Jan Jerabek ${ }^{*}$, and Jaroslav Koton ${ }^{*}$ \\ Dept. of Telecommunications ${ }^{*}$ / Radio Electronics ${ }^{\dagger}$, Brno University of Technology, Technicka 12, 61600 Brno, Czech Republic \\ ${ }^{\ddagger}$ Electronics and Communication Engineering Dept., Istanbul Technical University, 34469, Maslak, Istanbul, Turkey \\ Email: herencsn@feec.vutbr.cz
}

\begin{abstract}
In this paper, a fractional-order capacitor (FOC) of an order $\lambda=0.89$ (i.e. constant phase angle $-\mathbf{8 0 . 1}$ degree) was emulated via Valsa RC network with five branches. The network component values were optimized using modified least squares quadratic method in a wide frequency range of $100 \mathrm{mHz}-1 \mathrm{kHz}$ (i.e. 4 decades) and maximum relative phase error $0.78 \%$ was obtained. The design specification corresponds to a speed control system of an armature controlled DC motor, which is often used in control theory. Overall performance evaluation shows the product of evaluated key features (e.g. phase angle deviation and absolute values of relative phase, impedance, and pseudocapacitance errors) for the optimized FOC is $\mathbf{1 3 . 3 \%}$ less than the one obtained via Valsa approximation. The behavior of Op-Amp-based non-inverting configurations of analogue fractional-order integral operator $s^{-\lambda}$ employing the optimized FOC, where $0<\lambda<1$, is compared. The behavior of studied integrator circuits is confirmed by SPICE simulations using the readily available Texas Instruments TL072 low-noise Op-Amp macromodel, which is commonly used in electronics.
\end{abstract}

Keywords-DC motor, fractional calculus, fractional-order capacitor, fractional-order controller, fractional-order integrator, Op-Amp, Valsa RC network

\section{INTRODUCTION}

In the last decade, considerable attention was given to utilization of fractional calculus in various engineering disciplines. For instance, the control theory was stimulated by new analogue implementations of fractional-order (FO) proportional-integral-derivative (PID) controller design schemes. As it is known, FOPIDs are used for more than $90 \%$ of control applications in the industry, because the fractional calculus describes the dynamic characteristics of plant more precisely than integer-order description [1]. The survey [2] discusses the utilization of FO controllers in practice and draws the following conclusions:

- Potential reduction of the control effort, which also results in reduction of wasted energy, if FOPID controllers are applied to industrial problems.

- Heuristic FOPID tuning methods can be seen as attractive due to the relative simplicity of implementing the corresponding algorithms, but do suffer from issues related to the assessment of results.

Research described in this paper was financed by the Ministry of Education, Youth and Sports under grant LTC18022 of Inter-Cost program.
- An ideal, "basic building block" implementation of FO operators should be sought to compensate for the highorder approximation issue. This implementation must be cost effective to facilitate industrial adoption of FO controllers.

Fractional-order capacitors (FOCs) are key components for analogue FOPID design [3]-[7]. Different approaches for emulation of an FOC [8]-[13] and FO integral operator $s^{-\lambda}$ [14]-[16] are available in literature. Recursive algorithm by Valsa with its network structure, which is used in this work, is among them.

In brief, the paper is organized as follows: Section II describes preliminary considerations of a general control system and design specifications. Two different analogue implementations of $s^{-\lambda}$ of an order 0.89 employing Op-Amps are discussed within the same section. Simulation results of FOC computed via Valsa approximation and optimized using the modified least squares quadratic (MLSQ) method along with behavior of fractional-order integrators is shown in Section III, while the last section concludes the paper.

\section{CIRCUIT DESCRIPTION}

\section{A. General Description of a Control System}

A general block diagram of a single loop feedback control system is given in Fig. 1 and its transfer function (TF) can be expressed as:

$$
\frac{Y(s)}{R(s)}=\frac{C(s) G(s)}{1+C(s) G(s)}
$$

where $G(s)$ is a plant, $C(s)$ is a controller, $R(s)$ is a reference input signal, $Y(s)$ is an output signal, $T_{\mathrm{d}}(s)$ is an external disturbance, $U(s)$ is a control signal, and $E(s)$ is an error signal,

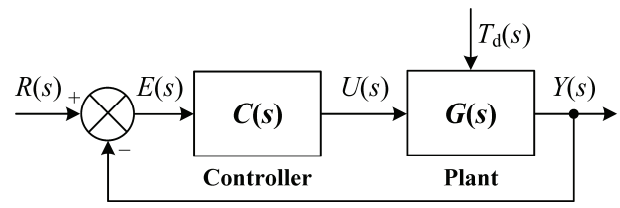

Fig. 1. Block diagram of a control system. 
which is given by $E(s)=R(s)-Y(s)$. An implementation of a control system used to control the speed and position of an armature controlled DC motor is shown in [16]. The system is composed of an analogue implementation of a FO proportional-integral (FOPI) controller $(C(s))$, while $G(s)$ is the mathematical model of a DC motor - the controlled plant [7]. Considering e.g. the setup [16], the speed of a DC motor can be controlled using FOPI, which TF has a form $C(s)=U(s) / E(s)=$ $K_{\mathrm{P}}+K_{\mathrm{I}} s^{-\lambda}$, where the particular three independent parameters are: $K_{\mathrm{P}}=1.37$ (proportional constant), $K_{\mathrm{I}}=2.28$ (integration constant), and $\lambda=0.89$ (fractional order of a non-inverting integrator in Laplace domain). Therefore, the next subchapter aims to compare the behavior of 0.89 -order integral operator implementations designed using two different techniques.

\section{B. Analogue Realizations of Fractional-Order Integral Operator $s^{-\lambda}$ Using Op-Amps}

Since late 1960s, the monolithic integrated circuit Op-Amp is one of the most versatile analog signal processing electronic components, which performs the basic mathematical operations, such as addition, subtraction, multiplication, and division. As it is known, it can also be used to do integration. Particular implementations of non-inverting integrators with two Op-Amps connected in inverted closed loop configuration (\#1) and single Op-Amp connected in non-inverted closed loop configuration (\#2) are shown in Fig. 2(a) and Fig. 2(b), respectively. Considering ideal Op-Amps that have the properties of infinite input resistance, zero output resistance, zero offset voltage, infinite frequency bandwidth, infinite common-mode rejection ratio, and most importantly infinite open-loop gain $A$, and for sake of simplicity assuming all resistors in circuits are matched, output voltages of integrators in time domain can be expressed as:

$$
v_{\text {OUT\#1 }}(t)=\frac{1}{C_{\lambda} R} \int_{0}^{t} v_{\mathrm{IN \# 1}}(t) d t^{\lambda}, v_{\text {OUT\#2 }}(t)=\frac{2}{C_{\lambda} R} \int_{0}^{t} v_{\mathrm{IN \# 2}}(t) d t^{\lambda},
$$

and equivalent TFs in Laplace domain can be given as:

$$
K_{\mathrm{I}^{\lambda} \# 1}(s)=\frac{V_{\mathrm{OUT} \# 1}(s)}{V_{\mathrm{IN \# 1}}(s)}=\frac{1}{s^{\lambda} C_{\lambda} R}, K_{\mathrm{I}^{\lambda} \# 2}(s)=\frac{V_{\mathrm{OUT} \# 2}(s)}{V_{\mathrm{IN} \# 2}(s)}=\frac{2}{s^{\lambda} C_{\lambda} R} .
$$

Here, the FOC with a pseudocapacitance $C_{\lambda}$ can be emulated via Valsa RC network shown in Fig. 3, which admittance in general form can be expressed as $Y_{C_{\lambda}}(s)=$ $=1 / R_{0}+s C_{0}+\sum_{k=1}^{n} s C_{k} /\left[s R_{k} C_{k}+1\right] \approx s^{\lambda} C_{\lambda}$. Now, to evaluate the gain and phase responses from (3), the $s^{\lambda}$ is replaced by $\omega^{\lambda}[\cos (\lambda \pi / 2)+j \sin (\lambda \pi / 2)]$ and the responses are given as:

$$
\begin{gathered}
\left|K_{\mathrm{I}^{\lambda} \# 1}(j \omega)\right|=\frac{1}{\omega^{\lambda} C_{\lambda} R},\left|K_{\mathrm{I}^{\lambda} \# 2}(j \omega)\right|=2\left|K_{\mathrm{I}^{\lambda} \# 1}(j \omega)\right|, \\
\varphi_{\mathrm{I}^{\lambda} \# 1}(j \omega)=\varphi_{\mathrm{I}^{\lambda} \# 2}(j \omega)=-\tan ^{-1}\left[\frac{\sin (\lambda \pi / 2)}{\cos (\lambda \pi / 2)}\right] .
\end{gathered}
$$

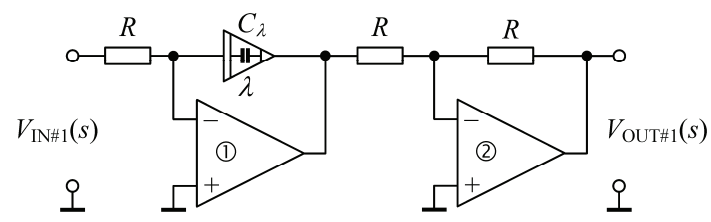

(a)

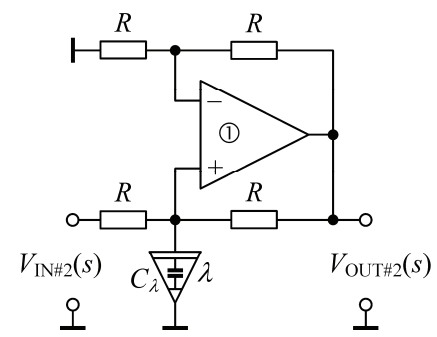

(b)

Fig. 2. Two implementations of fractional-order non-inverting integrator using Op-Amps: (a) configuration (\#1), (b) configuration (\#2).

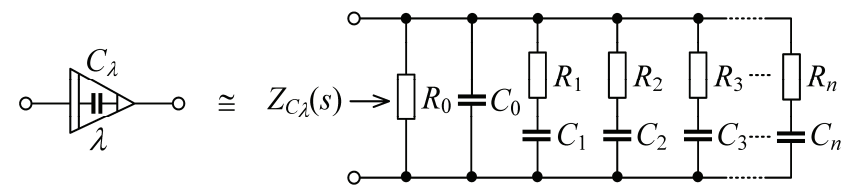

\begin{tabular}{|c|c|c|}
\hline \multirow{4}{*}{ \#1 } & $\begin{array}{c}K_{1^{\lambda} \# 1}(s) \\
\text { (a) }\end{array}$ & $\frac{A_{01} A_{02}}{\left(2+A_{02}\right)\left[1+s^{\lambda} C_{\lambda} R\left(1+A_{01}\right)\right]}$ \\
\hline & \multirow[b]{2}{*}{$\begin{array}{l}\left|K_{\mathrm{I}^{\lambda} \# 1}{ }^{\prime}(j \omega)\right| \\
\text { (b) }\end{array}$} & $A_{01} A_{02}$ \\
\hline & & $\left(2+A_{02}\right) \sqrt{\begin{array}{l}1+\omega^{2 \lambda} C_{\lambda}^{2} R^{2}\left(1+A_{01}\right)^{2}+ \\
+2 \omega^{\lambda} C_{\lambda} R\left(1+A_{01}\right) \cos (\lambda \pi / 2)\end{array}}$ \\
\hline & $\begin{array}{l}\varphi_{\mathrm{I}^{\lambda} \# 1}(j \omega) \\
\quad(\mathrm{c})\end{array}$ & $-\tan ^{-1}\left[\frac{\omega^{\lambda} C_{\lambda} R\left(1+A_{01}\right) \sin (\lambda \pi / 2)}{1+\omega^{\lambda} C_{\lambda} R\left(1+A_{01}\right) \cos (\lambda \pi / 2)}\right]$ \\
\hline \multirow{4}{*}{ \#2 } & $\begin{array}{c}K_{\mathrm{I}^{\lambda} \# 2}(s) \\
(\mathrm{d}) \\
\end{array}$ & $\frac{2 A_{01}}{4+s^{\lambda} C_{\lambda} R\left(2+A_{01}\right)}$ \\
\hline & \multirow[b]{2}{*}{$\left|K_{\mathrm{I}^{\lambda} \# 2}(j \omega)\right|$} & $2 A_{01}$ \\
\hline & & $\begin{array}{l}\sqrt{16+\omega^{2 \lambda} C_{\lambda}^{2} R^{2}\left(2+A_{01}\right)^{2}+} \\
+8 \omega^{\lambda} C_{\lambda} R\left(2+A_{01}\right) \cos (\lambda \pi / 2)\end{array}$ \\
\hline & $\begin{array}{c}\varphi_{\mathrm{I}^{\lambda} \# 2}{ }^{\prime}(j \omega) \\
\quad(\mathrm{f})\end{array}$ & $-\tan ^{-1}\left[\frac{\omega^{\lambda} C_{\lambda} R\left(2+A_{01}\right) \sin (\lambda \pi / 2)}{4+\omega^{\lambda} C_{\lambda} R\left(2+A_{01}\right) \cos (\lambda \pi / 2)}\right]$ \\
\hline
\end{tabular}

Fig. 3. Valsa RC network emulating fractional-order capacitor.

TABLE I. NON-IDEAL TFs, GAIN, AND PHASE EXPRESSIONS OF STUDIED INTEGRATORS.

In addition, the effect of finite open-loop gain of Op-Amps, which can be expressed as $A_{i}(s)=A_{0 i} /\left[1+s / \omega_{i}\right]$ for $i=\{1,2\}$, where $A_{0 i}$ denotes the dc gain and $\omega_{i}$ is the $-3-\mathrm{dB}$ frequency, was investigated. At low frequencies $A_{i}(s)=A_{0 i}$ and the resulted non-ideal expressions of both integrators in fractional domain are given in Table I.

\section{SimUlation RESUlts}

To verify the theoretical analysis, the behavior of the proposed FOC and studied integrators employing the readily available Texas Instruments TL072 low-noise Op-Amp macromodels have been simulated in SPICE software. The DC 


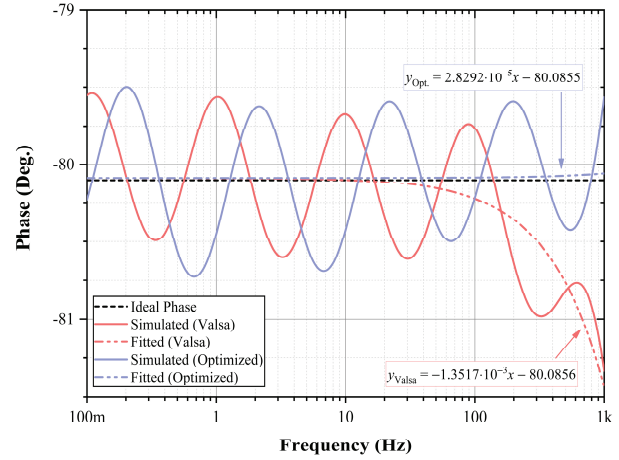

(a)

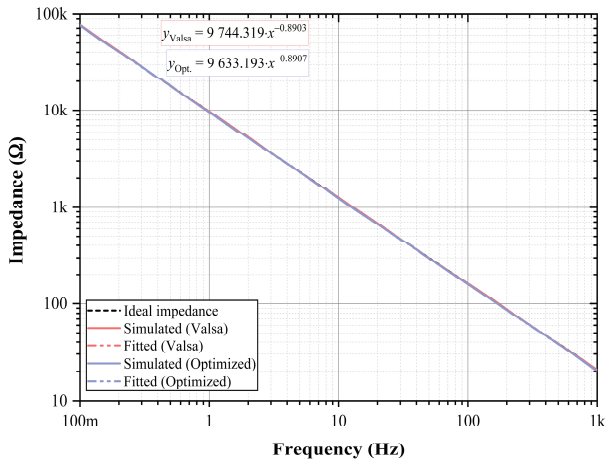

(b)

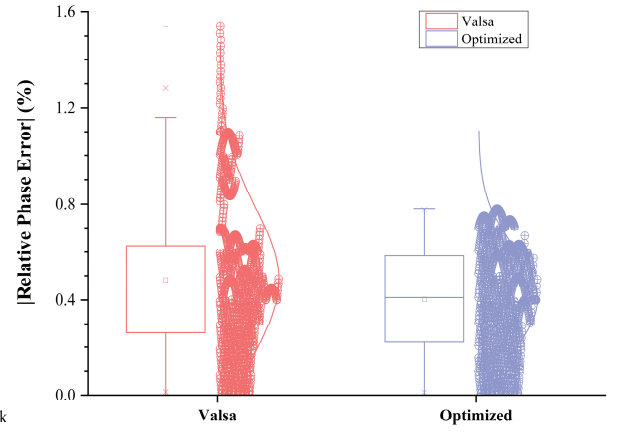

(c)

Fig. 5. Comparison of 0.89-order FOCs computed via Valsa approximation vs. its optimized realization: Ideal, simulated, and fitted (a) phase and (b) magnitude responses vs. frequency, (c) corresponding relative phase errors.

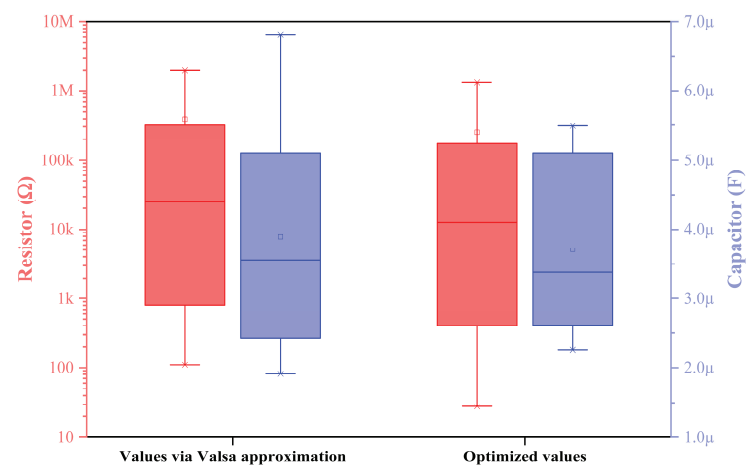

Fig. 4. Spread of resistor and capacitor values via Valsa approximation vs. its optimized values.

power supply voltages were equal to $\pm 5 \mathrm{~V}$. Firstly, the FOC with an order 0.89 and value $20 \mu \mathrm{F} \cdot \mathrm{s}^{-0.11}(12.7 \mu \mathrm{F} @ 10 \mathrm{~Hz})$, emulated via five branch Valsa RC network and optimized using MLSQ method, was designed for this purpose. The computed passive component values compliant with standard EIA-96 $( \pm 1 \%)$ are given in Table II. The total resistance/capacitance for both FOC realizations is investigated numerically, while their spread is depicted in Fig. 4. Comparative evaluation of both FOCs in terms of ideal, simulated, and fitted phase and magnitude responses versus frequency are given in Fig. 5 (a) and (b), respectively. The equivalent equations for fitting are provided in Figures as insets. Corresponding relative phase error analysis is depicted in Fig. 5(c). Figs. 4 and 5 indicate, the total resistance/capacitance and obtained relative phase error of optimized FOC is less than the one computed via Valsa approximation, while its frequency range is considerably wider $(100 \mathrm{mHz}-1 \mathrm{kHz}$, i.e. 4 decades). The overall performance evaluation is given in Table III, where key features, such as phase angle deviation and absolute values of relative phase, impedance, and pseudocapacitance errors are compared and depicted in Fig. 6 as a quadrilateral radar chart. Notably, the product of errors in case of the optimized FOC is $13.3 \%$ less than the one obtained via Valsa approximation. Secondly, the behavior of the studied non-inverting Op-Amp-based analogue realizations of fractional-order integrators employing the optimized FOC was evaluated. Resistor values in integrator
TABLE II. Standard EIA-96 COMPLIANT COMPONENT VALUES USED IN RC NETWORK DEPICTED IN FIG. 3 FOR $C_{0.89}=20 \mu \mathrm{F} \cdot \mathrm{s}^{-0.11} \mathrm{IN}$ FREQUENCY RANGE OF $100 \mathrm{mHz}-1 \mathrm{kHz}$.

\begin{tabular}{|c|c|c|}
\hline Components & $\begin{array}{c}\text { Values via Valsa } \\
\text { Approximation }\end{array}$ & $\begin{array}{c}\text { Optimized Values } \\
\text { via MLSQ method }\end{array}$ \\
\hline$R_{0}(\Omega) / C_{0}(\mathrm{~F})$ & $2 \mathrm{M} / 6.81 \mu$ & $1.3 \mathrm{M} / 5.49 \mu$ \\
\hline$R_{1}(\Omega) / C_{1}(\mathrm{~F})$ & $110 / 1.91 \mu$ & $27.4 / 2.61 \mu$ \\
\hline$R_{2}(\Omega) / C_{2}(\mathrm{~F})$ & $806 / 2.43 \mu$ & $402 / 2.26 \mu$ \\
\hline$R_{3}(\Omega) / C_{3}(\mathrm{~F})$ & $5.9 \mathrm{k} / 3.09 \mu$ & $2.87 \mathrm{k} / 2.94 \mu$ \\
\hline$R_{4}(\Omega) / C_{4}(\mathrm{~F})$ & $43.2 \mathrm{k} / 4.02 \mu$ & $22.1 \mathrm{k} / 3.83 \mu$ \\
\hline$R_{5}(\Omega) / C_{5}(\mathrm{~F})$ & $316 \mathrm{k} / 5.11 \mu$ & $174 \mathrm{k} / 5.11 \mu$ \\
\hline & Total resistance $(\Omega) /$ capacitance $(\boldsymbol{F})$ \\
\cline { 2 - 3 } & $2.37 \mathrm{M} / 23.37 \mu$ & $1.50 \mathrm{M} / 22.24 \mu$ \\
\cline { 2 - 3 }
\end{tabular}

TABLE III. COMPARISON OF SIMULATION RESUlTS OF DESIGNED FOCS IN FREQUENCY RANGE OF $100 \mathrm{mHz}-1 \mathrm{kHz}$.

\begin{tabular}{|c|c|c|}
\hline Evaluation Criteria & $\begin{array}{c}\text { FOC via Valsa } \\
\text { Approximation }\end{array}$ & $\begin{array}{c}\text { FOC Optimized } \\
\text { via MLSQ Method }\end{array}$ \\
\hline Phase angle deviation (Deg.) & 1.79 & 1.23 \\
\hline |Relative phase error| (\%) & 1.54 & 0.78 \\
\hline |Relative impedance error| (\%) & 1.79 & 2.79 \\
\hline |Relative pseudocap. error (\%) & 1.79 & 2.86 \\
\hline
\end{tabular}

|Relative phase error $\mid(\%) \quad$ Relative impedance error $\mid(\%)$

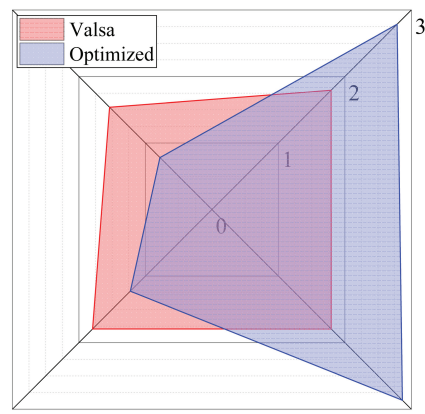

Phase angle deviation (Deg.) $\quad$ Relative pseudocap. error $(\%)$

Fig. 6. Radar chart showing an evaluation of FOCs computed via Valsa approximation vs. optimized realization from Table III. 


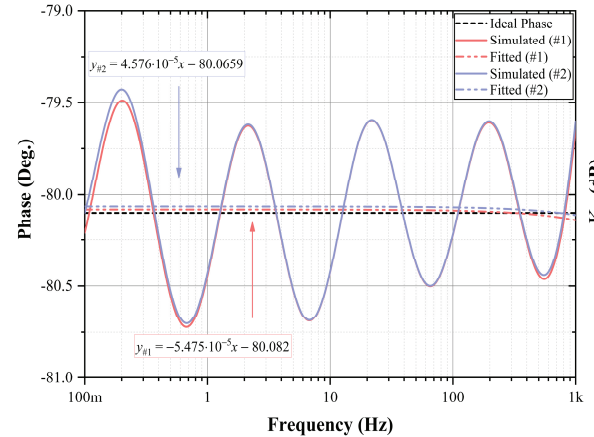

(a)

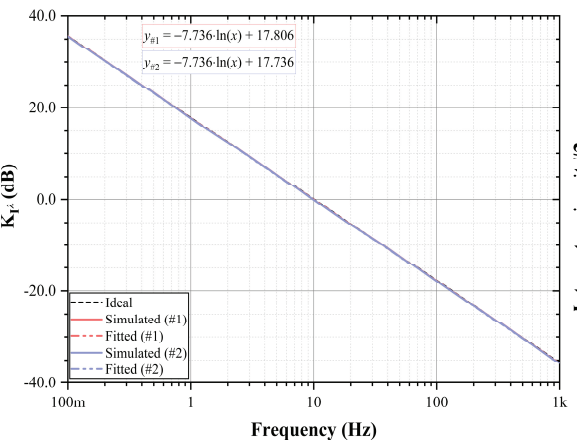

(b)

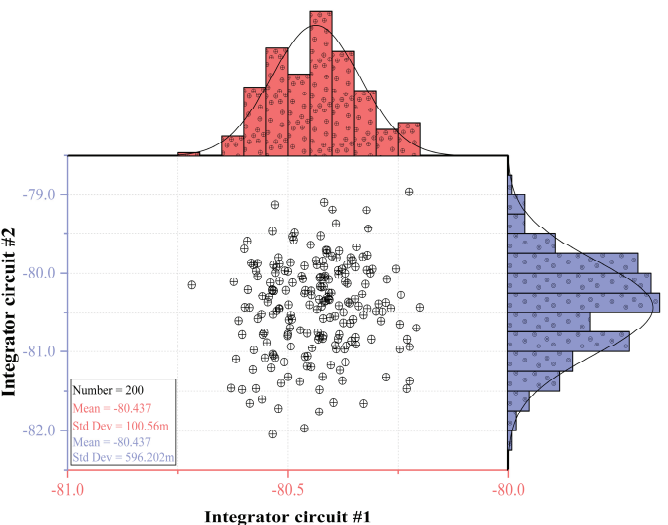

(c)

Fig. 7. Comparison of two realizations of 0.89-order integrators employing optimized FOC: Ideal, simulated, and fitted (a) phase and (b) gain responses vs. frequency, (c) Monte Carlo variation of the phase of $\mathrm{I}^{\lambda}$ at $10 \mathrm{~Hz}$.

circuits \#1 and \#2 were selected respectively as $R=1.24 \mathrm{k} \Omega$ and $R=2.49 \mathrm{k} \Omega$ in order to obtain unity-gain frequency of both integrators at $10 \mathrm{~Hz}$. Ideal, simulated, and fitted phase and gain responses of both 0.89 -order integrators in frequency domain are given in Fig. 7 (a) and (b), respectively. Equivalent equations for fitting the phase and gain via linear and natural logarithm regressions, respectively, are provided inside Figures. As it can be seen, in full frequency range of interest the phase angle deviation for \#1 and \#2 respectively are 1.23 and 1.27 degrees and corresponding relative phase errors are $0.78 \%$ and $0.83 \%$. Monte Carlo (statistical) analysis was performed with capacitors and resistors $1 \%$ tolerance and 200 runs with Gaussian distribution to observe effects of deviations due to manufacturing processes. The histogram shown in Fig. 7 (c) demonstrates the variation of the phase of both integrators at $10 \mathrm{~Hz}$. The mean and standard deviation values are given in Figure as an inset. Simulations confirmed that both investigated integrator structures show low sensitivity for deviations of passive components.

\section{CONCLUSION}

FOCs are key components for analogue controller design. Therefore, a FOC was emulated via Valsa RC network based on design specification that corresponds to a speed control system of an armature controlled DC motor. The FOC values were optimized (wide frequency range, low phase angle deviation as well as low maximum relative phase error) using MLSQ method and overall performance evaluation proves its better performance. The behavior of Op-Amp-based configurations of analogue fractional-order integral operator $s^{-\lambda}$ employing the optimized FOC is evaluated in detail.

\section{REFERENCES}

[1] J. Tenreiro-Machado, A. M. Lopes, D. Valério, and A. M. Galhano, Solved Problems in Dynamical Systems and Control. The IET, 2016.

[2] A. Tepljakov, B. B. Alagoz, C. Yeroglu, E. Gonzalez, S. H. HosseinNia, and E. Petlenkov, "FOPID controllers and their industrial applications: a survey of recent results," IFAC-PapersOnLine, vol. 51, pp. 25-30, 2018.

[3] I. Podlubny, B. Vinagre, P. O'leary, and L. Dorcak, "Analogue realizations of fractional-order controllers," Nonlinear Dynamics, vol. 29, pp. 281-296, 2002.
[4] A. Charef, "Analogue realisation of fractional-order integrator, differentiator and fractional $\mathrm{PI}^{\lambda} \mathrm{D}^{\mu}$ controller," IEE Proceedings Control Theory and Applications, vol. 153, no. 6, pp. 714-720, 2006.

[5] D. Sierociuk, I. Podlubny, and I. Petras, "Experimental Evidence of Variable-Order Behavior of Ladders and Nested Ladders," IEEE Trans. on Control Systems Technology, vol. 21, no. 2, pp. 459-466, 2013.

[6] H. Nezzari, A. Charef, and D. Boucherma, "Analog Circuit Implementation of Fractional Order Damped Sine and Cosine Functions," IEEE Journal on Emerging and Selected Topics in Circuits and Systems, vol. 3, no. 3, pp. 386-393, 2013.

[7] I. Petras, "Fractional-Order feedback control of a DC motor," Journal of Electrical Engineering, vol. 60, no. 3, pp. 117-128, 2009.

[8] A. M. Elshurafa, M. N. Almadhoun, K. N. Salama, and H. N. Alshareef, "Microscale electrostatic fractional capacitors using reduced graphene oxide percolated polymer composites," Applied Physics Letters, vol. 102, pp. 232901-232904, 2013.

[9] P. Ushakov, A. Shadrin, D. Kubanek, and J. Koton, "Passive fractionalorder components based on resistive-capacitive circuits with distributed parameters," in Proc. of 2016 39th Int. Conf. on Telecommunications and Signal Processing (TSP), Vienna, Austria, 2016, pp. 638-642.

[10] A. Kartci, A. Agambayev, N. Herencsar, and K. N. Salama, "Series-, parallel-, and inter-connection of solid-state arbitrary fractional-order capacitors: theoretical study and experimental verification," IEEE Access, vol. 6, pp. 10933-10943, 2018.

[11] J. Valsa, P. Dvorak, and M. Friedl, "Network model of the CPE," Radioengineering, vol. 20, pp. 619-626, 2011.

[12] G. Tsirimokou, A. Kartci, J. Koton, N. Herencsar, and C. Psychalinos, "Comparative study of discrete component realizations of fractionalorder capacitor and inductor active emulators," J. of Circuits Systems and Computers, vol. 27, no. 11, pp. 1850170-1-1850170-26, 2018.

[13] A. Kartci, A. Agambayev, M. Farhat, N. Herencsar, L. Brancik, H. Bagci, and K. N. Salama, "Synthesis and Optimization of FractionalOrder Elements Using a Genetic Algorithm," IEEE Access, vol. 7, pp. 1-14, 2019.

[14] R. Sotner, J. Jerabek, N. Herencsar, J. Petrzela, T. Dostal, and K. Vrba, "First-order adjustable transfer sections for synthesis suitable for special purposes in constant phase block approximation," AEU - Int. Journal of Electronics and Communications, vol. 69, pp. 1334-1345, 2015.

[15] R. Sotner, J. Jerabek, A. Kartci, O. Domansky, N. Herencsar, V. Kledrowetz, B. B. Alagoz, and C. Yeroglu, "Electronically Reconfigurable Two-Path Fractional-Order PI/D Controller Employing Constant Phase Blocks Based on Bilinear Segments Using CMOS Modified Current Differencing Unit," Microelectronics Journal, vol. 86, pp. 114-129, 2019.

[16] N. Herencsar, A. Kartci, J. Koton, R. Sotner, B. B. Alagoz, and C. Yeroglu, "Analogue Implementation of a Fractional-Order $\mathrm{PI}^{\lambda}$ Controller for DC Motor Speed Control," in Proc. of 2019 IEEE 28th Int. Symp. on Industrial Electronics (ISIE), Vancouver, Canada, in press, 2019, pp. 1-6. 